\title{
Dynamics of Ranking Processes in Complex Systems
}

\author{
Nicholas Blumm, ${ }^{1,2}$ Gourab Ghoshal, ${ }^{1,2,3}$ Zalán Forró, ${ }^{4}$ Maximilian Schich, ${ }^{1}$ Ginestra Bianconi, ${ }^{1}$ \\ Jean-Philippe Bouchaud, ${ }^{5}$ and Albert-László Barabási ${ }^{1,2}$ \\ ${ }^{1}$ Center for Complex Network Research, Department of Physics, Biology and Computer Science, Northeastern University, \\ Boston, Massachusetts 02115, USA \\ ${ }^{2}$ Department of Medicine, Harvard Medical School, and Center for Cancer Systems Biology, Dana-Farber Cancer Institute, \\ Boston, Massachusetts 02115, USA \\ ${ }^{3}$ The Media Laboratory, Massachusetts Institute of Technology, Cambridge, Massachusetts 02139, USA \\ ${ }^{4}$ Department of Management, Technology and Economics, ETH, CH-8032 Zurich, Switzerland \\ ${ }^{5}$ Capital Fund Management, 6 Boulevard Haussmann, 75009 Paris, France
}

(Received 6 April 2012; published 17 September 2012)

\begin{abstract}
The world is addicted to ranking: everything, from the reputation of scientists, journals, and universities to purchasing decisions is driven by measured or perceived differences between them. Here, we analyze empirical data capturing real time ranking in a number of systems, helping to identify the universal characteristics of ranking dynamics. We develop a continuum theory that not only predicts the stability of the ranking process, but shows that a noise-induced phase transition is at the heart of the observed differences in ranking regimes. The key parameters of the continuum theory can be explicitly measured from data, allowing us to predict and experimentally document the existence of three phases that govern ranking stability.
\end{abstract}

PACS numbers: 89.75.Hc, 64.60.al, 05.10.Gg, 05.40.-a

Some rankings, such as best-seller lists or trends measured by Twitter and Wikipedia, are intrinsically volatile, changing daily; others, such as intellectual achievement, show remarkable stability, keeping Darwin and Einstein as the most frequently mentioned scientists in the past 50 years [1-3]. These different patterns of ranking stability are illustrated in Fig. 1, which indicate that the has been the most frequently used English word in printed texts for at least 200 years [Fig. 1(a)]. In contrast, the ranking of research papers based on their citation patterns [Fig. 1(b)] displays significant volatility over time (see Fig. S10 for more examples [4]). While the items at the top of each list command high visibility, relatively little scientific attention is devoted to the rules and regularities of the ranking process itself. There is a good reason for this: rankings span such diverse areas, each with its own driving forces and peculiarities, that it appears to be a hopeless exercise to seek order in such a diverse subject. For example, the failures of existing models to capture the rank dynamics of cities [5] also indicate the need to address the dynamics of ranking across a wide range of data sets.

Yet, understanding the mechanisms that drive ranking raises a number of inherently deep questions: What distinguishes systems that display stable rankings from those whose ranking is inherently unstable, or volatile? Is the ranking a fair reflection of the intrinsic quality of the ranked items, or could lousy items also make it to the top?

Consider a list of items $i=1 \ldots N$, each assigned some score $X_{i}(t)$ that determines their ranking. We consider that in a given moment $t$ the item with the highest score $X_{i}(t)$ is ranked first $(r=1)$, and the one with the lowest score is ranked last $(r=N)$. Given that both the total number of items, $N$, and the total score, $\sum_{i=1}^{N} X_{i}(t)$, can vary with time, we focus on the normalized score $x_{i}(t)$, or the market share of item $i$,

$$
x_{i}(t)=\frac{X_{i}(t)}{\sum_{i} X_{i}(t)} .
$$

Note that, while an item's score may fluctuate around its mean value (exhibiting score stability), this does not guarantee its rank, as the rank is a relative measure of the score of all items in the system. Hence, the conditions required for score and rank stability are different.

In the data sets that we explore, the score $X_{i}$ represents the number of times individual words are used in published literature during a year [2], the daily market capitalization of companies [6], the number of diagnoses of a particular disease recorded in Medicare during a month [7], the number of annual citations each paper received in the Physical Review corpus $[1,8,9]$, the number of times a particular hashtag was used in tweets during a day [10,11], and the number of hourly page views of articles in Wikipedia [12] (Table S1). The temporal resolution of the data varies from hours to years and the time span from days to centuries.

The cumulative distribution of the market share of each item, $P(x)$, indicates that $x$ spans orders of magnitude (Fig. S11). In some systems, $P(x)$ follows a clear power law (word usage, Twitter), while in others we observe cutoffs at high $x_{i}$ (market cap, Medicare); $P(x)$ can be stationary or may shift in time. Despite these differences, changes in the market share of individual items follow a universal pattern [Figs. 1(c), 1(d), and S10e-h]. Indeed, we 
Class I: Stable ranking Word usage

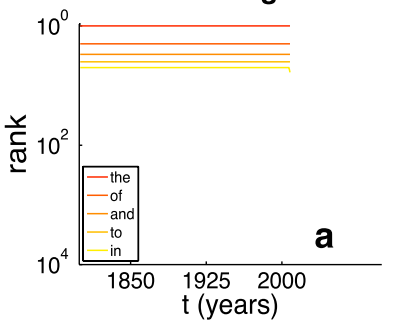

Class II: Unstable ranking Citation
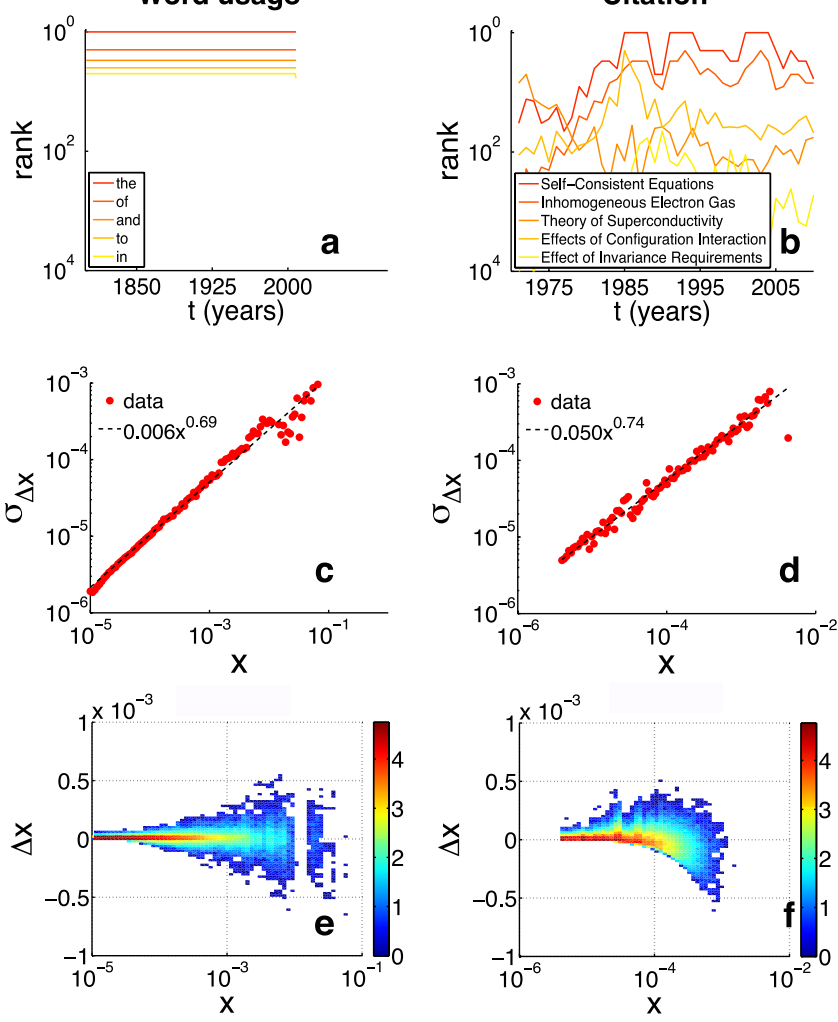

FIG. 1 (color online). Empirical characteristics of ranked systems. (a),(b) The evolution of the ranks of five top items in two systems: word usage displays ranking stability, and citation shows significant volatility. (c),(d) $\sigma_{\Delta x \mid x}$ in a function of $x$, indicating that for each system $\sigma_{\Delta x \mid x} \sim x^{\beta}$. (e),(f) Surface plot of $\Delta x$ in a function of $x$, indicating that for word usage $\Delta x$ fluctuates symmetrically around $\Delta x=0$, while we observe a remarkable asymmetry for high $x$ for citation. Similar data for four other systems, comparing company market capitalizations, disease diagnoses, Twitter hashtag usage, and Wikipedia page views, are shown in Fig. S10.

find that the dispersion $\sigma_{\Delta x \mid x}$ as a function of $x$-where $\Delta x$ is the change in an item's market share in a single time step-follows

$$
\sigma_{\Delta x \mid x} \sim x^{\beta},
$$

where $0.67 \leq \beta \leq 0.88$ (Table S1). The fact that $\beta<1$ implies that relative changes are typically smaller for topranked items, which is known in the economic context, where the volatility of large companies is less than that of small companies [6]. While such sublinear behavior $[13,14]$ may contribute to the stability of the high-ranked items, we do not detect any obvious correlations between $\beta$ and the observed ranking stability [Table S1 and Figs. 1(a) and 1(b)].

While (2) is universal, the nature and amplitude of the fluctuations appear to correlate with ranking stability. This is illustrated in Figs. 1(e), 1(f), and S10 where we show a scatter plot of $\Delta x$ in a function of $x$, indicating that, for word usage, Medicare, and market capitalization, $\Delta x$ fluctuates symmetrically around $\Delta x=0$; hence, the score of each item has comparable probability of moving up or down. In contrast citations, Wikipedia and Twitter display a remarkable asymmetry for high $x$, indicating that highscoring items have an enhanced tendency to drop in score.

To unveil the generic mechanisms that drive the ranking patterns, we simplify the system, assuming the score of each item follows the Langevin equation [15]

$$
\dot{x}_{i}=f\left(x_{i}\right)+g\left(x_{i}\right) \xi_{i}(t)-\phi(t) x_{i},
$$

where $f\left(x_{i}\right)$ represents the deterministic mechanisms that drive the score of item $i$, capturing a wide range of systemdependent attributes, from utility (words) to information content (Wikipedia) or impact (research papers), together with the relative fitness of each item compared to its peers.

The second term $g\left(x_{i}\right) \xi_{i}(t)$ captures the inherent randomness in the system; hence, $\xi_{i}(t)$ is a Gaussian random noise with $\left\langle\xi_{i}(t)\right\rangle=0$ and $\left\langle\xi_{i}(t) \xi_{i}\left(t^{\prime}\right)\right\rangle=\delta\left(t^{\prime}-t\right)$, and $g\left(x_{i}\right)$ models the noise amplitude, which may depend on the score $x_{i}$. The last term ensures that the scores are normalized, i.e., $\sum_{i} x_{i}(t) \equiv 1, \forall t$. Equation (3) assumes that the scores of different items do not directly influence each other.

We make two simplifying assumptions, suggested by empirical data. First, we postulate that the drift term $f\left(x_{i}\right)$ can be written as

$$
f\left(x_{i}\right)=A_{i} x_{i}^{\alpha},
$$

where $0<\alpha<1$ is identical for all $i$, whereas the coefficient $A_{i}$ can be interpreted as the "fitness" of item $i$ [16], capturing its intrinsic ability to increase its market share $x_{i}$. We also assume $g\left(x_{i}\right)$ has the form

$$
g\left(x_{i}\right)=B x_{i}^{\beta} .
$$

This choice is directly inspired by the measurements (2) [Figs. 1(c) and 1(d)], since the variance of score variations $\Delta x_{i}$ can be expressed as $\sigma_{\Delta x_{i} \mid x_{i}}^{2} \simeq g\left(x_{i}\right)^{2} \Delta t$ (see Eq. S5).

While $\beta$ is comparable for all systems, we observe significant differences in the magnitude of the coefficient $B$ : for systems with stable ranking, we find $B \approx 10^{-3}$, while, for the systems with unstable ranking, we observe $B \approx 10^{-2}-10^{-1}$ (Table S1). Since the coefficient $B$ is a direct measure of the noise magnitude, we find that the three unstable systems are affected by a higher level of noise than the three stable systems. Note that the difference between the two classes is independent of $\Delta t$ (see Sec. S3 and Fig. S12).

Next, we show that the observed differences in $B$ can induce a noise-driven phase transition in ranking, offering a quantitative explanation of the documented differences in ranking stability. Denoting by $P\left(x_{i}, t \mid A_{i}\right)$ the probability of an item to have score $x_{i}$ at time $t$ given its fitness $A_{i}$, the temporal evolution of $P\left(x_{i}, t \mid A_{i}\right)$ is governed by the Fokker-Planck equation 


$$
\frac{\partial P}{\partial t}=-\frac{\partial}{\partial x_{i}}\left\{\left[A_{i} x_{i}^{\alpha}-\phi(t) x_{i}\right] P\right\}+\frac{1}{2} \frac{\partial^{2}}{\partial x_{i}^{2}}\left(B^{2} x_{i}^{2 \beta} P\right) .
$$

Assuming that the system evolves towards a steady state with a time-independent $P_{0}\left(x_{i} \mid A_{i}\right)$ and a constant value for $\phi(t)=\phi_{0}$, the solution of (6) for $\alpha<1$ (Sec. S1.2) reads

$$
\begin{aligned}
P_{0}\left(x_{i} \mid A_{i}\right)= & C\left(A_{i}\right) x_{i}^{-2 \beta} \\
& \times \exp \left[\frac{2 A_{i}}{B^{2}} \frac{x_{i}^{1+\alpha-2 \beta}}{1+\alpha-2 \beta}\left\{1-\left(\frac{x_{i}}{x_{c}}\right)^{1 / \gamma}\right\}\right],
\end{aligned}
$$

where

$$
x_{c}=\left(\frac{A_{i}}{\phi_{0}}\right)^{\gamma}\left(\frac{2(1-\beta)}{1+\alpha-2 \beta}\right)^{\gamma} \quad \text { with } \quad \gamma \equiv \frac{1}{1-\alpha},
$$

and $C\left(A_{i}\right)$ is the normalization constant.

To find the most probable value of $x_{i}$, we set the derivative of Eq. (7) to zero, obtaining

$$
F\left(x_{i}\right)=\left(A x_{i}^{\alpha}-\phi_{0} x_{i}\right)-\frac{B^{2}}{2} \beta x_{i}^{2 \beta-1}=0 .
$$

For $B=0$, the solution of Eq. (9) is

$$
x_{i}^{\star}=\left(\frac{A_{i}}{\phi_{0}}\right)^{\gamma} \text {. }
$$

One can obtain Eq. (10) directly from the steady-state solution of Eq. (3) with $f\left(x_{i}\right)$ defined in Eq. (4) and $g\left(x_{i}\right)=0$. Equation (10) indicates that each item will converge to a score determined by its fitness $A_{i}$ and a combination of all other fitnesses via $\phi_{0}=\left(\sum_{i} A_{i}^{\gamma}\right)^{1 / \gamma}$.

In the presence of noise, Eq. (9) indicates that $B$ shifts the steady-state value of $x_{i}$ from its deterministic solution $x_{i}^{\star}$ to a new $x_{i}^{\star}+\delta_{i}$. Equation (9) predicts that changes in the noise magnitude $B$ induce a dynamical phase transition from a stable (confined score) to an unstable (delocalized score) phase. For $B<B_{c}$, Eq. (9) has two solutions, at $x_{i}=x_{i}^{\star}+\delta_{i}$ (stable) and $x_{i}=0$ (unstable) (Fig. S13b). Therefore, $P\left(x_{i} \mid A_{i}\right)$ is unimodal with a sharp peak (Fig. S13e), indicating that for low noise the market share of item $i$ will be localized around a value determined by the interplay between the item's fitness and the noise magnitude. At $B=B_{c}$, the nonzero solution disappears $\left(\left(x_{i}=x_{i}^{\star}+\delta_{i} \rightarrow 0\right.\right.$; see Figs. S13c and S13f). Equation (7) predicts that for $B>B_{c}$ the distribution follows a power law $P\left(x_{i} \mid A_{i}\right) \sim x_{i}^{-2 \beta}$ with an exponential cutoff at high $x_{i}$. This implies that $x_{i}$ is no longer confined to the vicinity of $x_{i}^{\star}$ but becomes delocalized, varying over orders of magnitude.

The consequence of the predicted noise-induced phase transition is illustrated in Figs. S13h-j, where we show the evolution of the ranks for the top five fitness items, as predicted by numerical simulations of Eq. (3). In the stable phase $\left(B<B_{c}\right)$, the top items maintain their nominal rank, determined by their respective fitnesses, similar to the behavior observed in Fig. 1(a). At the critical point
$B=B_{c}$, the stable ranking is perturbed by unstable bursts, an intermittent behavior common in dynamical systems at the critical point $[17,18]$. Finally, for $B>B_{c}$ most items become delocalized, lacking rank stability, a behavior similar to the one observed in Fig. 1(b).

To further test the predictive power of our model in Fig. S13, we plot the average change in score $\langle\Delta x\rangle$ and $\sigma_{\Delta x \mid x}$ as a function of the score $x$. For $B<B_{c}$, the score variation $\Delta x$ fluctuates symmetrically around $\Delta x=0$, similar to the behavior observed in stable systems [Fig. 1(e)]. For $B>B_{c}$, however, there is a systematic downward trend for high $x$, as observed for the unstable systems [Fig. 1(f)]. Indeed, while a precise empirical determination of the drift term is difficult, the choice of its functional form Eq. (4) is supported by the qualitative agreement between Figs. 1(e), 1(f), S13k, and S13m.

Score stability, the expectation that $x_{i}$ fluctuates around a well-defined mean, is a necessary but not a sufficient condition for an item to maintain a stable rank. Rank is a collective measure: it depends not only on the score $x_{i}$ of item $i$, but also the score of all other items $x_{j}(j \neq i)$ to which $x_{i}$ is compared. While an item may be score-stable, its fluctuation around $x_{i}^{\star}$ may be sufficiently large so that $P_{0}\left(x_{i} \mid A_{i}\right)$ for items with comparable $x_{i}^{\star}$ may overlap and thus the items can swap ranks, a phenomenon akin to the melting of a solid, where atoms cannot remain in register due to thermal agitation. Score (and rank) stability can occur only in the $B<B_{c}$ regime, and a saddle point approximation provides the magnitude of the fluctuations in $x_{i}$ around its steady-state market share $x_{i}^{\star}$ (see Sec. S2.1). Assuming that the intrinsic fitnesses $A_{i}$ are drawn from $P(A) \sim A^{-(1+\mu)}$ (see Fig. S11 for empirical support), we can relate the rank fluctuation of an item to its rank $r$ (Eq. S21 and [9]):

$$
\sigma_{r}=\left(\frac{\gamma}{2 \phi_{0}}\right)^{1 / 2} B\langle x\rangle_{r}^{\beta}
$$

For an item to show rank stability, the inequality $\Delta_{r}=$ $\langle x\rangle_{r}-\langle x\rangle_{r+1}>\sigma_{r}$ predicts a second critical value of the noise coefficient, $B_{r}<B_{c}$, controlling rank stability (where $\left\langle x_{r}\right\rangle$ is the expected score of an item with rank $r$ ).

These results indicate that the stability of a list is best captured by the $A-B$ phase diagram shown in Fig. 2(a). The top part $\left(B>B_{c}\right)$ is the unstable region, where Eq. (7) predicts that the score of each item is broadly distributed; hence, neither rank nor score stability is possible, similar to a gas phase where atoms move at random. In the region $B<B_{c}$, we have score stability, which means that each score fluctuates around a steady-state market share $x^{\star}$ determined by its intrinsic fitness. Yet, score stability does not necessarily imply rank stability. Hence, there are two distinct phases below $B_{c}$ : a score-stable (liquid) phase between $B_{c}$ and $B_{r}$, where each item has a stable score but the fluctuations around $x^{\star}$ are sufficient for items with comparable score to swap rank. For $B$ below $B_{r}$, we find a solid phase: the noise is sufficiently low so that items 

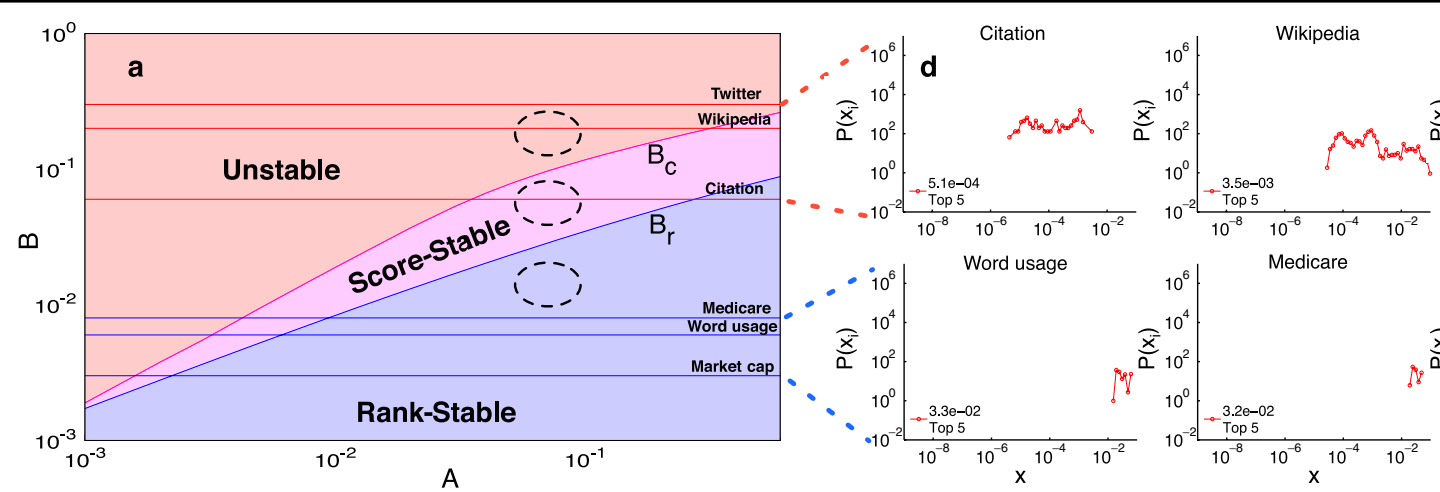

Twitter
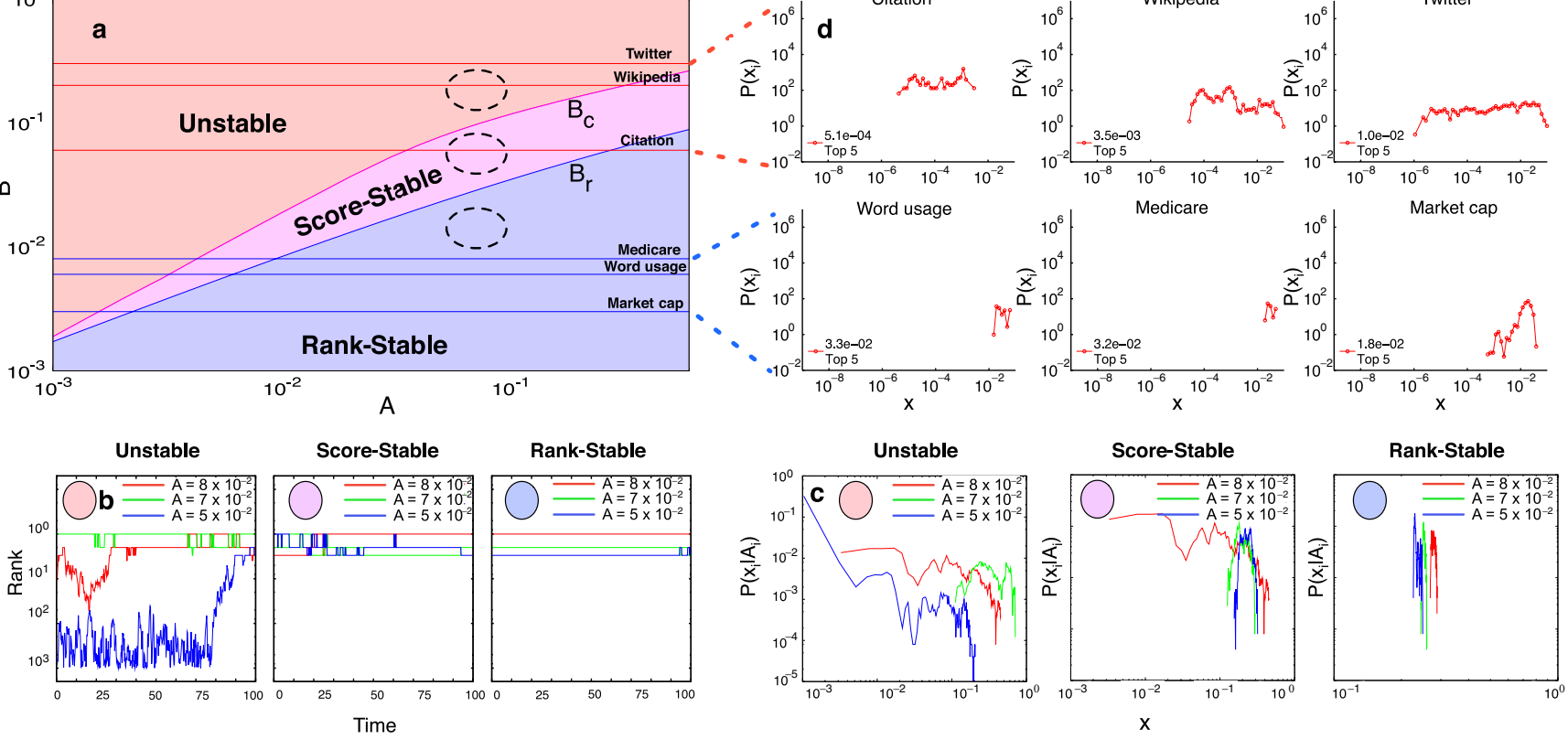

FIG. 2 (color online). Characterization of the phases in ranking stability. (a) The $A-B$ phase diagram of ranking stability calculated by numerically solving Eq. (9). The $B_{c}$ and $B_{r}$ lines correspond to the phase boundaries calculated in the text. (b) Time evolution of the ranks for the top-fitness items in each of the three predicted phases. (c) The market share distributions $P\left(x_{i} \mid A_{i}\right)$ for the top ranks in the three predicted phases calculated by integrating (3) numerically. (d) Market share distribution $P\left(x_{i}\right)$ for the items with the highest market share $x$ in each of the studied real systems. We find that, for the three stable systems, $P\left(x_{i}\right)$ for the top items is distributed narrowly in the high $x$ region, spanning less than an order of magnitude, in line with the predictions for the stable phase. For the other three systems, the market share of the top-ranked items varies over 3-4 orders of magnitude, in line with the fat tailed form (9) predicted for this region.

display not only score but also rank stability. These predictions are supported by Fig. 2(b), where we plot the time evolution of ranks for the top items in each phase.

In the phase diagram of Fig. 2(a), we can represent each real system with a horizontal line placed at its experimentally measured $B$ (Table S1). Medicare, word usage, and market cap are deep in the rank-stable regime, indicating that highly ranked items should display rank stability, a prediction that agrees with the empirical results of Figs. 1(a) and S10. An order of magnitude gap in $B$ separates them from the three unstable systems, Twitter, Wikipedia, and citation, that are deeply in the unstable phase.

Our theory predicts that for score-stable systems the market share $x_{i}$ of the high-fitness items should be narrowly distributed, in contrast with the unstable phase, where $x_{i}$ fluctuates widely [Fig. 2(c)]. We therefore measured $P\left(x_{i}\right)$ for top-scoring items in each system [Fig. 2(d)], finding that, as expected, in the three stable systems $P\left(x_{i}\right)$ is narrow, spanning less than an order of magnitude. In contrast, for the three unstable systems $P\left(x_{i}\right)$ spans 3-4 orders of magnitude.

Taken together, our results indicate that the ranking stability observed in some systems and the volatile ranking observed in others represent different phases separated by a noise-induced phase transition. Despite the diversity of the explored systems, their dynamics can be qualitatively captured by a single continuum theory that predicts the existence of three dynamically distinct phases. In analogy with critical phenomena, one could view $B$ as the control parameter and the score's standard deviation as the order parameter.

With suitable enrichments-for example, through the incorporation of fads and herding [19,20]—the introduced formalism may be extendible to a wider range of systems such as biodiversity and linguistics [21,22]. A detailed account should also consider the role of memory and correlations (see Secs. S6 and S7 for results on this). In our formalism, we take into account global shocks [23], since $\phi(t)$ enforces normalization. Local correlations, present for related items, could reduce the relative motion between items, increasing rank stability. Such stability could shift the rank-stable transition upwards in Fig. 2. Remarkably, the model qualitatively emulates correlations observed in the data even without explicitly enforcing local correlations (Figs. S1 and S2).

[1] F. Radicchi, S. Fortunato, B. Markines, and A. Vespignani, Phys. Rev. E 80, 056103 (2009).

[2] J.-B. Michel et al., Science 331, 176 (2011).

[3] J. Bohannon, Science 331, 143 (2011). 
[4] See Supplemental Material at http://link.aps.org/ supplemental/10.1103/PhysRevLett.109.128701 for additional details on both the model and the data.

[5] M. Batty, Nature (London) 444, 592 (2006).

[6] R. Mantegna and H. Stanley, Nature (London) 376, 46 (1995).

[7] C. Hidalgo, N. Blumm, A. Barabási, and N. Christakis, PLoS Comput. Biol. 5, e1000353 (2009).

[8] P. Chen, H. Xie, S. Maslov, and S. Redner, J. Informetrics 1, 8 (2007).

[9] G. Ghoshal and A. Barabási, Nature Commun. 2, 394 (2011).

[10] S. Golder and M. Macy, Science 333, 1878 (2011).

[11] M. Cha, H. Haddadi, F. Benevenuto, and K. Gummadi, in Proceedings of the 4th International AAAI Conference on Weblogs and Social Media (ICWSM), 2010, http://citeseerx .ist.psu.edu/viewdoc/summary?doi=10.1.1.167.192.

[12] A. Capocci, F. Rao, and G. Caldarelli, Europhys. Lett. 81, 28006 (2008).
[13] M. de Menezes and A. Barabási, Phys. Rev. Lett. 92, 028701 (2004).

[14] Z. Eisler, I. Bartos, and J. Kertész, Adv. Phys. 57, 89 (2008).

[15] J. Bouchaud and M. Mézard, Physica (Amsterdam) 282A, 536 (2000).

[16] G. Bianconi and A. Barabási, Europhys. Lett. 54, 436 (2001).

[17] D. Brockmann and T. Geisel, Phys. Rev. Lett. 90, 170601 (2003).

[18] S. Dorogovtsev, A. Goltsev, and J. Mendes, Rev. Mod. Phys. 80, 1275 (2008).

[19] M. Salganik, P. Dodds, and D. Watts, Science 311, 854 (2006).

[20] C. Borghesi and J. Bouchaud, Quality and quantity 41, 557 (2007).

[21] I. Volkov, J. Banavar, S. Hubbell, and A. Maritan, Nature (London) 424, 1035 (2003).

[22] R. F. i Cancho and R. Solé, Proc. R. Soc. B 268, 2261 (2001).

[23] D. Sornette, F. Deschatres, T. Gilbert, and Y. Ageon, Phys. Rev. Lett. 93, 228701 (2004). 